\title{
Analysis and Simulations of Dynamic Models of Hepatitis B Virus
}

\author{
Xisong Dong (Corresponding author) \\ National Engineering Laboratory for Disaster Backup and Recovery \\ Beijing University of Posts and Telecommunications \\ 10 Xi Tu Cheng Road, Beijing 100876, China
}

$\&$

The Key Laboratory of Complex Systems and Intelligence Science

Institute of Automation, Chinese Academy of Sciences

95 Zhong Guan Cun East Road, Beijing 100190, China

Tel: 86-10-6255-4288 E-mail: dongcomic04@163.com

Cong Wang

National Engineering Laboratory for Disaster Backup and Recovery

Beijing University of Posts and Telecommunications

10 Xi Tu Cheng Road, Beijing 100876, China

Tel: 86-10-6228-3366 E-mail: wangc@bupt.edu.cn

Gang Xiong

The Key Laboratory of Complex Systems and Intelligence Science

Institute of Automation, Chinese Academy of Sciences

95 Zhong Guan Cun East Road, Beijing 100190, China

Tel: 86-10-6255-4288 E-mail: gang.xiong@ia.ac.cn

The research is financed by Beijing Natural Science Foundation, China (No. 4092029)

\begin{abstract}
A mathematical model consisting of two systems of Hepatitis B virus (HBV) infection is set up based on the model of virus dynamics and experimental observation of anti-viral drug therapy for HBV infection patients provided by Nowak. A mathematical analysis of dynamic behaviors shows that the model has two kinds of equilibrium points, which represent the patients' complete recovery and HBV persistent infection at the end of the treatment with drug lamivudine, respectively. Our model may provide possible quantitative interpretations for the long-time treatments of chronic HBV infections with the drug lamivudine according to different model parameters, in particularly it may explain why the plasma virus of Nowak et al's patients turnover the original level after stopping the lamivudine treatment.
\end{abstract}

Keywords: Hepatitis B virus(HBV), Mathematical model, Lamivudine treatment, Numerical simulation

\section{Introduction}

Hepatitis B is a potentially life-threatening liver infection caused by the hepatitis B virus (HBV). It is a major global health problem and the most serious type of viral hepatitis. It is a viral infection that attacks the liver and can cause both acute and chronic disease (WHO, 2002). About 2 billion people worldwide have been infected with the virus and about 350 million live with chronic infection. An estimated 600000 persons die each year due to the acute or chronic consequences of hepatitis B (WHO, 2010). About $25 \%$ of adults who become chronically infected during childhood later die from liver cancer or cirrhosis (scarring of the liver) caused by the chronic infection. HBV is 50 to 100 times more infectious than HIV (WHO, 2010). HBV is an important occupational hazard for health workers, and 50 million new cases are diagnosed annually (WHO, 2010).

Recently drugs called interferon or lamivudine have been used to treat patients with chronic hepatitis B. Considering the need for various long-term treatments, it is necessary to construct a mathematical model that enables us to study the dynamics of HBV (Moskovitz, 2005; Nowak, 2000).

In (Nowak, 2000), 95 patients were treated with various dose of lamivudine for 28 days or 24 weeks respectively. Based on the clinical data, Nowak proposed a mathematical model with three variables: uninfected cells, infected cells, and free 
virus (Nowak, 2000). This model can describe some viral dynamics in HBV infection. But, the model did not analyzed mathematically, and the model cannot describe the phenomena that the plasma virus of most patients resurged rapidly as soon as the drug was withdrawn.

In this paper, a mathematical model consisting of two systems is designed. One system is the model proposed by Nowak which describes the viral dynamics in HBV infection after withdrawing the lamivudine therapy. The other system is a model with four state variables: uninfected cells, infected cells, free virus and cytotoxic cells, which represent the viral dynamics in HBV infection during the lamivudine therapy. The mathematical analysis of the dynamics of our model shows that the two systems have two kinds of equilibrium points, which represent the patient's complete recovery and HBV persistent infection, respectively. And the numerical simulation can explain why the plasma virus of Nowak et al's patients turnover the original level after stopping the lamivudine treatment, and it also may predict the long-time treatments of chronic HBV infections with the drug lamivudine according to different model parameters,.

\section{Immune Model of HBV Infection and Analysis}

\subsection{The HBV Model}

we propose the following mathematical model to describe the viral dynamics of the anti-HBV infection treatment with lamivudine.

During processing the lamivadine therapy, we assume that the immune model of HBV infection has the form:

$$
\left\{\begin{array}{l}
\frac{\mathrm{d} x}{\mathrm{~d} t}=\lambda-d x-b x v \\
\frac{\mathrm{d} y}{\mathrm{~d} t}=b x v-a y-k_{3} y \\
\frac{\mathrm{d} v}{\mathrm{~d} t}=k y-u v-k_{1} y \\
\frac{\mathrm{d} e}{\mathrm{~d} t}=k_{1} y-k_{2} e
\end{array}\right.
$$

where the 4 variables- $x, y, v$ and $e$ represent the numbers of uninfected cells, infected cells, free virus, and cytotoxic cells, respectively. $\lambda$ is the rate of reproduced susceptible cells. Uninfected cells die at rate $d x$, and become infect at rate $b x v$, where $b$ is the rate constant describing the infection process. Infected cells are produced at rate $b x v$ and die at rate $a y$. Free virus are produced from infected cells at rate $k y$ and removed at rate $u v . k_{1}, k_{3}$ represent the pharmacological effect of the lamivadine to infected cells and free virus, respectively. Cytotoxic cells are produced at rate $k_{1} y$ and removed at rate $k_{2} e . \lambda, d, b, a, k, u, k_{1}, k_{2}, k_{3}$ are positive constant and will be determined by antiviral immune responses.

After withdrawing the lamivadine therapy, the dynamic model of HBV infection is still described by Nowak's model at (Nowak, 2000):

$$
\left\{\begin{array}{l}
\frac{\mathrm{d} x}{\mathrm{~d} t}=\lambda-d x-b v x \\
\frac{\mathrm{d} y}{\mathrm{~d} t}=b v x-a y \\
\frac{\mathrm{d} v}{\mathrm{~d} t}=k y-u v
\end{array}\right.
$$

$\mathrm{Eq}(1)$ has two equilibrium points:

$$
\begin{aligned}
Q_{1}^{*} & =\left(\frac{\lambda}{d}, 0,0,0\right), \\
Q_{2}^{*} & =\left(x^{*}, \frac{\lambda-d x^{*}}{a+k_{3}}, \frac{\lambda-d x^{*}}{b x^{*}}, \frac{k_{1}\left(\lambda-d x^{*}\right)}{k_{2}\left(a+k_{3}\right)}\right) .
\end{aligned}
$$

where

$$
x^{*}=\frac{u\left(a+k_{3}\right)}{b\left(k-k_{1}\right)} .
$$

$\mathrm{Eq}(2)$ has two equilibrium points:

$$
\begin{aligned}
Q_{1} & =\left(\frac{\lambda}{d}, 0,0\right), \\
Q_{2} & =\left(\frac{a u}{b k}, \frac{d}{a}\left(\frac{\lambda}{d}-\frac{a u}{b k}\right), \frac{\lambda}{b}\left(\frac{b k}{a u}-\frac{d}{\lambda}\right)\right) .
\end{aligned}
$$

Clearly, the equilibrium points $Q_{1}, Q_{1}^{*}$ and $Q_{2}, Q_{2}^{*}$ stand for the patient's complete recovery and HBV persistent infection, respectively. 
2.2 Analysis of Equilibrium Points

\section{Lemma 1:}

$$
\begin{aligned}
\mathbf{a} & =\left[a_{0}, a_{1}, \cdots, a_{n-1}\right], \\
p(s, \mathbf{a}) & =s^{n}+a_{n-1} s^{n-1}+\cdots+a_{1} s+a_{0}, \\
\hbar_{n} & =\left\{\mathbf{a} \in \mathbb{R}^{n}: p(s, \mathbf{a})=0 \Rightarrow \operatorname{Re}[s]<0\right\}, \\
\hbar_{3} & =\left\{\mathbf{a} \in \mathbb{R}^{3}: a_{1} a_{2}>a_{0}, a_{i}>0, i=0,1,2\right\}, \\
\hbar_{4} & =\left\{\mathbf{a} \in \mathbb{R}^{4}: a_{1} a_{2} a_{3}>a_{1}^{2}+a_{0} a_{3}^{2}, a_{i}>0, i=0,1,2,3\right\} .
\end{aligned}
$$

Lemma 2: Denote

$$
\dot{\mathbf{X}}=f(\mathbf{X})
$$

where

$$
\begin{aligned}
\mathbf{X} & =\left(x_{1}, x_{2}, \cdots, x_{n}\right) \in \mathbb{R}^{n}, \\
f(\mathbf{X}) & =\left(f_{1}(\mathbf{X}), f_{2}(\mathbf{X}), \cdots, f_{n}(\mathbf{X})\right)^{\mathrm{T}}, \\
A\left(\mathbf{X}^{*}\right) & =\left[\begin{array}{ccc}
\frac{\partial f_{1}(\mathbf{X})}{\partial x_{1}} & \cdots & \frac{\partial f_{1}(\mathbf{X})}{\partial x_{n}} \\
\vdots & \ddots & \vdots \\
\frac{\partial f_{n}(\mathbf{X})}{\partial x_{1}} & \cdots & \frac{\partial f_{n}(\mathbf{X})}{\partial x_{n}}
\end{array}\right]_{\mathbf{X}=\mathbf{X}^{*}}
\end{aligned}
$$

If $f\left(\mathbf{X}^{*}\right)=0$ and $f$ is differentiable on $\mathbf{X}^{*}$,

1. If $\forall \lambda \in \sigma(A) \Rightarrow \operatorname{Re}(\lambda)<0$, then $\mathbf{X}^{*}$ is an asymptotically stable equilibrium point;

2. If $\forall \lambda \in \sigma(A) \Rightarrow \operatorname{Re}(\lambda)>0$, then $\mathbf{X}^{*}$ is an unstable equilibrium point.

Where $\sigma(A)$ are the eigenvalues of $A\left(\mathbf{X}^{*}\right)$.

2.2.1 Analysis of Equilibrium Points $Q_{1}, Q_{2}$

Case 1: If the parameters satisfy the inequality:

$$
A=\frac{\lambda b k}{a d u}<1,
$$

then the equilibrium point $Q_{1}$ is an asymptotically stable equilibrium point because all the eigenvalues of the linearized equation of $\mathrm{Eq}(2)$ at $Q_{1}$

$$
\begin{aligned}
& \lambda_{1}=-d, \\
& \lambda_{2}=\frac{-(a+u)+\left[(a+u)^{2}+4\left(\frac{\lambda b k}{d}-a u\right)\right]^{\frac{1}{2}}}{2}, \\
& \lambda_{3}=\frac{-(a+u)-\left[(a+u)^{2}+4\left(\frac{\lambda b k}{d}-a u\right)\right]^{\frac{1}{2}}}{2},
\end{aligned}
$$

are less than 0 .

For another equilibrium point $Q_{2}$, it can be proved that the eigenfunction of the Jacobean matrix of the linearized equation of $\mathrm{Eq}(2)$ at $Q_{2}$ has the form

$$
\begin{aligned}
p(s, \mathbf{a}) & =s^{3}+\frac{a^{2} u+a u^{2}+\lambda b k}{a u} s^{2}+\frac{a b k \lambda+b k u \lambda}{a u} s+\lambda b k-a d u \\
& \triangleq s^{3}+a_{2} s^{2}+a_{1} s+a_{0}
\end{aligned}
$$

Because

$$
a_{0}=\lambda b k-a d u<0,
$$

from Lemma $1, p(s, \mathbf{a})=0$ has a root with positive real part. This means that $Q_{2}$ is unstable. 
Case 2: If the parameters satisfies the inequality

$$
A=\frac{\lambda b k}{a d u}>1,
$$

then the equilibrium point $Q_{1}$ is unstable equilibrium point because one of the real parts of the eigenvalues of the Jacobian matrix of the linearized equation of $\mathrm{Eq}(2)$ at $Q_{1}$ is larger than 0 . On the other hand, we can get

$$
a_{i}>0, i=0,1,2, \quad a_{1} a_{2}>\lambda b k>a_{0} .
$$

From Lemma 1 and 2, we have $Q_{2}$ is asymptotically stable.

2.3.2 Analysis of Equilibrium Points $Q_{1}^{*}, Q_{2}^{*}$

Case 1: If the parameters satisfies the inequality

$$
A^{*}=\frac{\lambda b\left(k-k_{1}\right)}{\left(a+k_{3}\right) d u}<1
$$

then the equilibrium point $Q_{1}^{*}$ is an asymptotically stable equilibrium point because all the eigenvalues of the Jacobian matrix of the linearized equation of $\operatorname{Eq}(1)$ at $Q_{1}^{*}$

$$
\begin{aligned}
& \lambda_{1}=-d, \\
& \lambda_{2}=-k_{2}, \\
& \lambda_{3}=\frac{-\left(a+u+k_{3}\right)+\left[\left(a+u+k_{3}\right)^{2}+4\left(\frac{\lambda b\left(k-k_{1}\right)}{d}-\left(a+k_{3}\right) u\right)\right]^{\frac{1}{2}}}{2}, \\
& \lambda_{4}=\frac{-\left(a+u+k_{3}\right)-\left[\left(a+u+k_{3}\right)^{2}+4\left(\frac{\lambda b\left(k-k_{1}\right)}{d}-\left(a+k_{3}\right) u\right)\right]^{\frac{1}{2}}}{2},
\end{aligned}
$$

are less than 0 .

For another equilibrium point $Q_{2}^{*}$, it can be proved that the eigenequation of the Jacobean matrix of the linearized equation of $\mathrm{Eq}(2)$ at $Q_{2}^{*}$ has the form

$$
\begin{aligned}
p(s, \mathbf{a}) & =s^{4}+\frac{\lambda k_{2}+q\left(u+a+k_{2}+k_{3}\right)}{q} s^{3}+\frac{\left(\lambda\left(k_{2}+u+a\right)+q\right)\left(a+u+k_{3}\right)}{q} s^{2}+\frac{\left(\lambda k_{2}+u-d u q\right)\left(u+k_{3}\right)}{q} s \\
& +\frac{u k_{2}\left(u+k_{3}\right)(\lambda-d q)}{q} \triangleq s^{4}+a_{3} s^{3}+a_{2} s^{2}+a_{1} s+a_{0},
\end{aligned}
$$

where

$$
q=\frac{\left(a+k_{3}\right) u}{b\left(k-k_{1}\right)}
$$

Because

$$
a_{0}=\frac{u k_{2}\left(u+k_{3}\right)(\lambda-d q)}{q}=\frac{u k_{2}\left(u+k_{3}\right)}{q}\left(\lambda-d \frac{\left(a+k_{3}\right) u}{b\left(k-k_{1}\right)}\right)=\frac{u k_{2} \lambda\left(u+k_{3}\right)}{q}\left(1-\frac{1}{A^{*}}\right)<0
$$

then from Lemma 1 and 2, $Q_{2}^{*}$ is unstable.

Case 2: If the parameters satisfies the inequality

$$
A^{*}=\frac{\lambda b\left(k-k_{1}\right)}{\left(a+k_{3}\right) d u}>1
$$

then the equilibrium point $Q_{1}^{*}$ is unstable because one of the real parts of the eigenvalues of the Jacobean matrix of the linearized equation of $\mathrm{Eq}(2)$ at $Q_{1}^{*}$ is larger than 0 . On the other hand, we can get

$$
\begin{gathered}
A^{*}>1 \Rightarrow k>k_{1} \Rightarrow q>0 \Rightarrow a_{i}>0, i=1,2,3, \\
\lambda-d q=\lambda-\frac{\left(a+k_{3}\right) d u}{b\left(k-k_{1}\right)}=\lambda\left(1-\frac{1}{A^{*}}\right)>0 \Rightarrow a_{0}>0,
\end{gathered}
$$




$$
\begin{aligned}
a_{1} a_{2} a_{3} & -\left(a_{1}^{2}+a_{0} a_{3}^{2}\right) \\
& =\frac{\left(\lambda^{2}\left(k_{3}+u+a\right)+\lambda\left(q a^{2}+u^{2}+q\left(a+k_{3}\right) u\right)+d q^{2}\left(a+k_{3}\right) u\right)\left(\left(a+k_{3}\right)\left(q k_{2}^{2}+k_{2} \lambda+u(\lambda-d q)\right)+k_{2}^{2} u q+k_{2}^{3} q+k_{2}^{2} \lambda\right)}{q^{3}} \\
& >\frac{\left(\lambda^{2}\left(k_{3}+u+a\right)+\lambda\left(q a^{2}+u^{2}+q\left(a+k_{3}\right) u\right)+d q^{2}\left(a+k_{3}\right) u\right)\left(\left(a+k_{3}\right)\left(q k_{2}^{2}+k_{2} \lambda\right)+k_{2}^{2} u q+k_{2}^{3} q+k_{2}^{2} \lambda\right)}{q^{3}} \\
& >0
\end{aligned}
$$

From Lemma 1 and 2, we can get $Q_{2}^{*}$ is asymptotically stable.

Because

$$
A=\frac{\lambda b k}{a d u}<1 \Rightarrow A^{*}=\frac{\lambda b\left(k-k_{1}\right)}{\left(a+k_{3}\right) d u}<1
$$

then when

$$
A=\frac{\lambda b k}{a d u}<1,
$$

$Q_{1}, Q_{1}^{*}$ are both asymptotically stable equilibrium points; because

$$
A^{*}=\frac{\lambda b\left(k-k_{1}\right)}{\left(a+k_{3}\right) d u}>1 \Rightarrow A=\frac{\lambda b k}{a d u}>1
$$

then when

$$
A^{*}=\frac{\lambda b\left(k-k_{1}\right)}{\left(a+k_{3}\right) d u}>1,
$$

$Q_{2}, Q_{2}^{*}$ are both unstable.

\section{Numeric simulation}

Now let us use our model $(\mathrm{Eq}(1)$ and (2)) to simulate the antiviral treatment of chronic HBV infection with drug lamivadine reported by Nowak(Nowak, 2000) using MATLAB program.

Take $\left\{a, d, u, k_{1}, \lambda\right\}=\left\{0.053,0.053,0.67,0.05,8.48 \times 10^{6}\right\}$ and different values of other parameters and initial values, the simulation result is displayed in Figure 1.

From Figure 1, it can be concluded that our model may provide possible quantitative interpretations for the treatment of chronic HBV infection with the drug lamivudine reported by Nowak(Nowak, 2000), in particularly explain why the plasma virus of patients turnover the original level after stopping the lamivudine therapy.

If we prolong the treatment time, we can get Figure2.

The values of patient 1-6 for criteria of the stabilities of equilibrium points are listed in Table 1:

From Table 1, we can see that for the patient 1,3,5, $Q_{1}, Q_{1}^{*}$ is unstable equilibrium point and $Q_{2}, Q_{2}^{*}$ is stable one and for the patient $2,4,6, Q_{2}, Q_{2}^{*}$ is stable and $Q_{1}, Q_{1}^{*}$ is unstable. So, if we prolong treatment time, Patient 2,4,6 may recovered completely, and Patient 1,3,5 may hardly be cured even we prolong the time of therapy.

In summary, we can obtain following conclusions:

(1). If inequality (3) and (4) holds, prolonging treatment time makes $v\left(t^{*}\right)<0$ at some time $t^{*}$ then the patient can recovered completely. In fact, it has been found that if prolonging patient 4's treatment time from 28 days to about 200 days, the virus can be deleted (see Figure 2).

(2). If inequalities (3) or (4) do not hold, then the necessity of treatment with drug lamivadine seems to be questionable. Although the population of infected cells and virus are reduced, and the population of uninfected cells is increased remarkably, prolonging treatment time may not make the patient recovered. As soon as withdrawn treatment of drug, the population of infected cells, uninfected cells and free virus will return the level before therapy (see Figure 1).

\section{Conclusion}

In this paper, a mathematical model consisting of two systems of Hepatitis B virus (HBV) infection is set up which has two kinds of equilibrium points, representing the patients' complete recovery and HBV persistent infection, respectively. Our model can explain why the plasma virus of Nowak et al's patients turnover the original level after stopping the lamivudine treatment, and it may provide possible quantitative interpretations for the long-time treatments of chronic HBV infections with the drug lamivudine according to different model parameters.

Some parameters of models of HBV infection are difficult (if not impossible) to be obtained directly. However, theoretical analysis may be helpful for figure out them. Practically, the dynamic behaviors of HBV infection are very complex and puzzling. Our research might provide a possible interpretation for some of them. More and accurate experimental 
data are needed for modeling dynamics of HBV infection. It seems that new treatment approaches are expected to treat patients. The quantitative understanding of HBV dynamics will make it possible to devise optimal treatment strategies for individual patients. Further research for HBV dynamics is promising.

\section{References}

Lee, W. (1997). Hepatitis B virus infection, New Eng. J. Med. 337, 1733-1745.

Moskovitz, N. D., Osiowy, C. \& Giles E. et al. (2005). Response to long-term lamivudine treatment(up to 5 years)in patients with severe chronic hepatitis B,role of genotype and drug resistance, J.of Viral Hepatitis, 12, 398-403.

Nowak, M. A. \& Robert, M. (2000). Virus dynamics-mathematical principles of immunology and virology. Oxford university press. 2000.

World Health Organization. (2002). Department of Communicable Disease Surveillance and Response Hepatitis B. [Online] Available: http://www.who.int/csr/disease/hepatitis/HepatitisB_whocdscsrlyo2002_2.pdf.

WHO2010. (2010). Hepatitis B. [Online] Available: http://www.who.int/mediacentre/factsheets/fs204/en/ (June 10, 2010).

Table 1. The dynamic values of patients

Patient 1 Patient 2 Patient 3 Patient 4 Patient 5 Patient 6

\begin{tabular}{lllllll}
\hline & & & & & & \\
$A$ & 23.008 & 9.0000 & 21.000 & 26.000 & 26.000 & 9.000 \\
$A^{*}$ & 6.0157 & 0.2757 & 1.0647 & 0.3271 & 1.3182 & 0.4563 \\
\hline
\end{tabular}
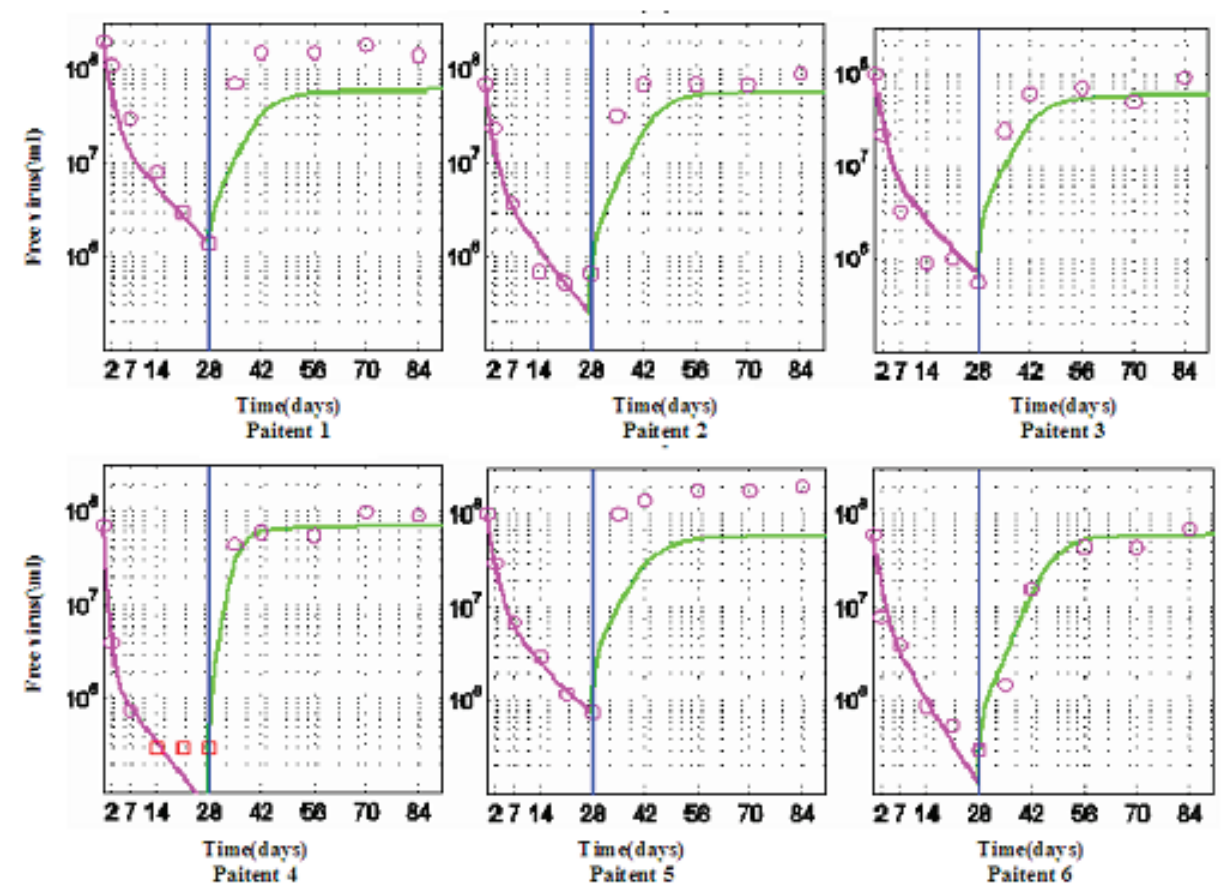

Figure 1. Dynamic routs of improved HBV immune model(the circles are the numbers reported by Nowak 

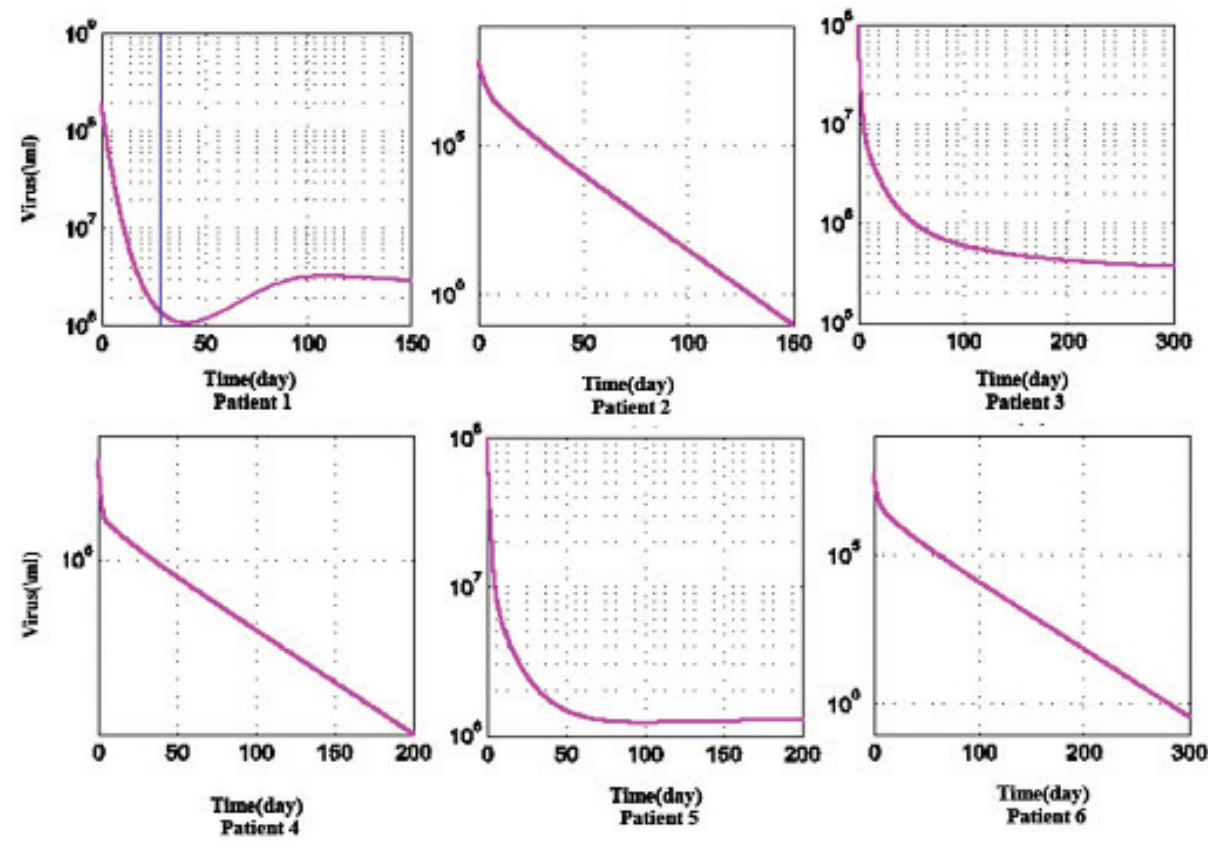

Figure 2. Long-Time dynamic routs of improved HBV immune model 